\title{
A note on integral representation of Feller kernels
}

\author{
by R. RȨBOWski (Wrocław)
}

\begin{abstract}
We consider integral representations of Feller probability kernels from a Tikhonov space $X$ into a Hausdorff space $Y$ by continuous functions from $X$ into $Y$. From the existence of such a representation for every kernel it follows that the space $X$ has to be 0 -dimensional. Moreover, both types of representations coincide in the metrizable case when in addition $X$ is compact and $Y$ is complete. It is also proved that the representation of a single kernel is equivalent to the existence of some non-direct product measure on the product space $Y^{\mathbb{N}}$.
\end{abstract}

Introduction. Let $X$ and $Y$ be Hausdorff spaces and let $\mathcal{B}_{Y}$ be the Borel $\sigma$-algebra in $Y$. A Feller kernel $p$ on $X \times \mathcal{B}_{Y}$ is a continuous mapping $x \rightarrow p(x, \cdot)$ from $X$ into the space of all Radon probabilities on $Y$ endowed with the weak* topology. The set of all Feller kernels on $X \times \mathcal{B}_{Y}$ will be denoted by $\Phi$.

The space $C(X, Y)$ of all continuous functions from $X$ into $Y$ can be embedded as a subspace of $\Phi$. Indeed, every $\varphi$ in $C(X, Y)$ defines the deterministic Feller kernel $p_{\varphi}(x, A)=1_{A}(\varphi(x))$. It is obvious that $\Phi$ is convex and $p_{\varphi}$ is an extreme point of $\Phi$ for every $\varphi$ in $C(X, Y)$. If in addition $X$ is separable metrizable and $Y$ is Polish then the extreme points of $\Phi$ are exactly the deterministic Feller kernels (see [4] for details).

We endow $\Phi$ with the least $\sigma$-algebra for which all the mappings $p \rightarrow$ $p(x, A)\left(x \in X, A \in \mathcal{B}_{Y}\right)$ are measurable. In $C(X, Y)$ we define the least $\sigma$-algebra $\Sigma$ for which the embedding $\varphi \rightarrow p_{\varphi}$ is measurable. In other words, $\Sigma$ is the least $\sigma$-algebra which makes measurable all the evaluation mappings $\varphi \rightarrow \varphi(x)(x \in X)$.

We say that the Feller kernel $p \in \Phi$ has an integral representation on $\Sigma$ if there exists a probability measure $\mu$ on $\Sigma$ such that

$$
p(x, A)=\int p_{\varphi}(x, A) d \mu(\varphi) \quad\left(x \in X, A \in \mathcal{B}_{Y}\right) .
$$

Equivalently, $p(x, \cdot)=\pi_{x}(\mu)$ where $\pi_{x}$ is the evaluation map $\pi_{x}(\varphi)=\varphi(x)$

1991 Mathematics Subject Classification: Primary 60J35; Secondary 28C20.

Key words and phrases: Feller kernel, integral representation. 
on $C(X, Y)$. The above formula gives a Choquet-type integral representation for $p \in \Phi$.

In $C(X, Y)$ we can also consider the $\sigma$-algebra $\mathcal{C}$ of Borel sets for the compact-open topology in $C(X, Y)$. Clearly $\Sigma \subset \mathcal{C}$.

The integral representation problem for Feller kernels has been considered by Blumenthal and Corson in [1,2] (see also [3]-[5]). In [1] they proved the following theorem:

Let $X$ be a 0-dimensional compact Hausdorff space and let $Y$ be complete metrizable. Then for every Feller kernel $p$ on $X \times \mathcal{B}_{Y}$ there is a Radon measure $\mu$ on $\mathcal{C}$ such that $p(x, \cdot)=\pi_{x}(\mu)$ for all $x$ in $X$.

Hence if $X$ and $Y$ satisfy the assumptions of the above theorem, the existence of the integral representation on $\Sigma$ also follows for every $p \in \Phi$.

In Section 1 we show that the 0-dimensionality assumption on $X$ is in fact necessary in the Blumenthal and Corson theorem and we prove that the representation of every $p \in \Phi$ by means of a Radon measure on $\mathcal{C}$ is in fact equivalent to the integral representation on $\Sigma$ for every $p \in \Phi$ under rather mild conditions on $X$ and $Y$.

Section 2 shows that the existence of an integral representation on $\Sigma$ for a single Feller kernel is equivalent to the existence of a certain non-direct product measure on $Y^{\mathbb{N}}$.

1. Necessary conditions for integral representation. We begin by showing that the 0-dimensionality assumption on $X$ in the BlumenthalCorson integral representation theorem is in fact necessary. This makes precise a remark in [1], p. 194.

Indeed, assume that $X$ is a Tikhonov space and $Y$ is a Hausdorff space containing at least two points. We prove that if every Feller kernel $p$ on $X \times \mathcal{B}_{Y}$ has an integral representation by a Radon measure $\mu$ on $\mathcal{C}$ then $X$ is 0 -dimensional. To this end, take an open neighbourhood $U$ of $x_{0}$ in $X$. Without loss of generality we may assume $U \neq X$. Fix a continuous function $g$ from $X$ into the unit interval such that $g\left(x_{0}\right)=1$ and $g(x)=0$ on $X \backslash U$. Then $x_{0} \in Z(1-g) \subset U$ and $Z(g) \cap Z(1-g)=\emptyset$, where $Z(h)$ denotes the zero set of $h$.

For any two different points $y$ and $z$ in $Y$ we define a Feller kernel $p$ by

$$
p(x, \cdot)=g(x) \delta_{y}+(1-g(x)) \delta_{z}
$$

and take a probability Radon measure $\mu$ on $\mathcal{C}$ which represents $p$. Now, since $\mu$ is Radon, we have $\mu(\{\varphi: \varphi(X) \subset\{y, z\}\})=1$ and clearly $\mu(\{\varphi: \varphi(x)=$ $y\})=1$ on $Z(1-g)$ while $\mu(\{\varphi: \varphi(x)=z\})=1$ on $Z(g)$. Hence there is a mapping $\varphi \in C(X, Y)$ such that $\varphi(Z(g))=\{z\}, \varphi(Z(1-g))=\{y\}$ and $\varphi(X)=\{y, z\}$. This gives a partition of $X$ into two closed-and-open sets $V$, 
$W$ such that $Z(1-g) \subset V$ and $Z(g) \subset W$. Finally, since $x_{0} \in V \subset U$, we see that $X$ is 0 -dimensional.

In general $\Sigma \neq \mathcal{C}$, so there is no reason for the measure $\mu$ on $\Sigma$ which represents $p \in \Phi$ to have an extension to some Radon measure on the larger $\sigma$-algebra $\mathcal{C}$. Nevertheless, we have a similar result for integral representation on $\Sigma$ under an additional separability condition.

TheOREM 1. Let $X$ be a separable metrizable space and let $Y$ be Hausdorff with at least two elements. If every Feller kernel on $X \times \mathcal{B}_{Y}$ has an integral representation on $\Sigma$ then $X$ is 0-dimensional.

Proof. Let $g$ and $p$ be as in the above proof and assume that $p$ has an integral representation on $\Sigma$. By using, instead of the Radon property, the fact that $X, Z(g)$ and $Z(1-g)$ are separable, we obtain as before $\varphi(X)=$ $\{y, z\}, \varphi(Z(1-g))=\{y\}$ and $\varphi(Z(g))=\{z\}$ for some $\varphi \in C(X, Y)$. This yields the 0-dimensionality of $X$.

Now by combining the Blumenthal-Corson theorem and Theorem 1 we have

Corollary. Let $X$ and $Y$ be metric spaces with $X$ compact and $Y$ complete. Assume $Y$ has at least two elements. Then the following conditions are equivalent:

(1) $X$ is 0-dimensional.

(2) Every $p \in \Phi$ has an integral representation on $\mathcal{C}$ by a Radon measure.

(3) Every $p \in \Phi$ has an integral representation on $\Sigma$.

2. Integral representation of Feller kernels. Let $X$ be an infinite separable Hausdorff space and let $Y$ be metrizable. For every $\varphi \in C(X, Y)$ let $T \varphi=\left(\varphi\left(x_{1}\right), \varphi\left(x_{2}\right), \ldots\right) \in Y^{\mathbb{N}}$, where $\left\{x_{n}\right\}$ is a fixed dense subset of $X$ with $x_{i} \neq x_{j}$ for $i \neq j$. Then $T$ is 1-1 but need not be onto $Y^{\mathbb{N}}$ and we denote by $\operatorname{im}(T)$ the image of $C(X, Y)$ in $Y^{\mathbb{N}}$ under $T$. It is easy to check that $T^{-1}\left(\mathcal{B}_{Y^{\mathbb{N}}}\right)=\Sigma$, where $\mathcal{B}_{Y^{\mathbb{N}}}$ denotes the Borel $\sigma$-algebra in $Y^{\mathbb{N}}$ endowed with the product topology.

The last observation allows us to give an alternative description of the representing measure in terms of a non-direct product measure on $\mathcal{B}_{Y^{\mathbb{N}}}$.

THeOREm 2. Let $X$ be an infinite separable Hausdorff space and let $Y$ be metrizable. For every Feller kernel $p$ on $X \times \mathcal{B}_{Y}$ the following conditions are equivalent:

(1) $p$ has an integral representation on $\Sigma$.

(2) There exists a probability measure $\lambda$ on $\mathcal{B}_{Y^{\mathbb{N}}}$ with $n$-th marginal $\lambda_{n}$ equal to $p\left(x_{n}, \cdot\right)$ and the outer measure $\lambda^{*}(\operatorname{im} T)$ equal to one. 
Proof. $(1) \Rightarrow(2)$. The equality $\Sigma=T^{-1}\left(\mathcal{B}_{Y^{\mathbb{N}}}\right)$ implies $\lambda^{*}(\operatorname{im} T)=1$ for $\lambda:=T(\mu)$. Since for every $n=1,2, \ldots$ and $A \in \mathcal{B}_{Y}$ we have $\lambda_{n}(A)=$ $(T(\mu))_{n}(A)=p\left(x_{n}, A\right)$, the condition (2) is satisfied.

$(2) \Rightarrow(1)$. Note that the condition $\lambda^{*}(\operatorname{im} T)=1$ allows us to define a probability measure $\mu$ on $\Sigma$ such that $T(\mu)=\lambda$. In particular, for every Borel set $A$ in $Y$ and every $n$ we have $\mu\left(\left\{\varphi: \varphi\left(x_{n}\right) \in A\right\}\right)=\lambda_{n}(A)=$ $p\left(x_{n}, A\right)$. Fix $x_{0} \in X$ and choose a sequence $z_{n} \rightarrow x_{0}$ selected from $\left\{x_{n}\right\}$. For any nonempty closed subset $F$ in $Y$ define $V_{n}=\{y: d(y, F)<1 / n\}$ and $F_{n}=\{y: d(y, F) \leq 1 / n\}$ where $d(y, F)$ is the distance of $y$ from $F$. Since for every open (closed) set $A$ the function $x \rightarrow p(x, A)$ is lower (upper) semicontinuous, the Fatou lemma implies

$$
\begin{gathered}
\int 1_{F}\left(\varphi\left(x_{0}\right)\right) d \mu(\varphi) \leq \int 1_{V_{n}}\left(\varphi\left(x_{0}\right)\right) d \mu(\varphi) \leq \int \liminf _{k} 1_{V_{n}}\left(\varphi\left(z_{k}\right)\right) d \mu(\varphi) \\
\leq \underset{k}{\limsup } \int 1_{V_{n}}\left(\varphi\left(z_{k}\right)\right) d \mu(\varphi) \leq \limsup _{k} p\left(z_{k}, F_{n}\right) \leq p\left(x_{0}, F_{n}\right)
\end{gathered}
$$

for every $n$. Consequently, for every closed set $F$ in $Y$ and every $x$ in $X$ we have $\int 1_{F}(\varphi(x)) d \mu(\varphi) \leq p(x, F)$. Since the left hand side is a probability measure on the metric space $Y$, this implies that $\mu$ in fact represents $p$.

\section{References}

[1] R. M. Blumenthal and H. H. Corson, On continuous collections of measures, Ann. Inst. Fourier (Grenoble) 20 (2) (1970), 193-199.

[2] - - - On continuous collections of measures, in: Proc. of the Sixth Berkeley Sympos. on Math. Statistics and Probability, Vol. II, Berkeley and Los Angeles, Univ. of Calif. Press, 1972, 33-40.

[3] N. Ghoussoub, An integral representation of randomized probabilities and its applications, in: Séminaire de Probabilités XVI, Lecture Notes in Math. 920, Springer, Berlin 1982, 519-543.

[4] A. Iw anik, Integral representations of stochastic kernels, in: Aspects of Positivity in Functional Analysis, R. Nagel, U. Schlotterbeck and M. P. H. Wolff (eds.), Elsevier, 1986, 223-230.

[5] Y. Kifer, Ergodic Theory of Random Transformations, Progr. Probab. Statist. 10, Birkhäuser, Boston 1986.

INSTITUTE OF MATHEMATICS

TECHNICAL UNIVERSITY OF WROCEAW

WYBRZEŻE WYSPIAŃSKIEGO 27

50-370 WROCŁAW, POLAND 